\title{
ANOS POTENCIAIS DE VIDA PERDIDOS SEGUNDO CAUSAS, EM FORTALEZA (BRASIL), 1978-80*
}

\author{
Marcelo Gurgel Carlos da Silva**
}

\begin{abstract}
SILVA, M.G.C. da Anos potenciais de vida perdidos segundo causas, em Fortaleza (Brasil), 1978-80. Rev. Saúde públ., S. Paulo, 18: 108 -21, 1984.
\end{abstract}

RESUMO: Estudo realizado para determinar e analisar os anos potenciais de vida perdidos (APVP) das principais causas de óbitos, segundo sexo e idade, em Fortaleza, em 1978-80. Os óbitos foram distribuídos segundo causa, sexo e idade. A técnica adotada para os APVP foi a sugerida por Romeder e McWhinnie, tendo sido definida a idade limite de vida potencial em 65 anos. Os resultados mostraram que o total de APVP foi 197.942,5, nos homens, e 137.545, nas mulheres, correspondendo a taxas de APVP de 119,82 por 1.000 homens e 73,06 por 1.000 mulheres, e que as violências, com 41,15 por 1.000 homens e 8,46 por 1.000 mulheres, foram as maiores responsáveis pelos APVP, nos dois sexos, arcando com $34,35 \%$ dos APVP no masculino e 11,57\% no feminino. Em ordem de importancia em APVP, as principais causas foram as violências, as doenças infecciosas intestinais, as outras doenças do aparelho respiratório, as doenças do aparelho digestivo e o câncer, nos homens, $e$ as violências, as doenças do aparelho respiratório, o câncer, as doenças infecciosas intestinais e as doenças cerebrovascula. res, nas mulheres. Foi ressaltada a importância da utilização da metodologia dos APVP para o planejamento em saúde, como instrumento de orientação à hierarquização de prioridades.

UNITERMOS: Mortalidade. Indicadores de saúde. Planejamento em saúde.

\section{INTRODUÇÃO}

Os indicadores de saúde são os parâmetros empregados na mensuração das condi. ções de saúde da população, na definição de escalas de prioridades e na avaliação dos recursos, sendo, portanto, de grande relevância no planejamento e administração de programas.

Com a preocupação de definir e medir o "nivel de vida", a Organização das Nações Unidas, em 1952, convocou um grupo de trabalho para elaborar as variáveis que deveriam ser observadas para a caracterização dos padrões de vida. Por sua vez, a Organização Mundial de Saúde, de acordo com as recomendações da $\mathrm{ONU}$, designou um grupo de peritos para analisar os indicadores sugeridos ao componente "Saúde, incluindo condiçðes demográficas" 914 .

A análise da mortalidade é de especial importância no campo da demografia da saúde e, neste, as estatísticas de óbitos representam, praticamente, o único instrumento para medir o nível de saúde da comunidade e para a elaboração e a avaliação dos programas de saúde 4 .

Presentemente, outros indicadores têm sido propostos, e, dentre esses, vem recebendo maiores atenções o estudo dos anos potenciais de vida perdidos.

Esse estudo vem sendo trabalhado segun-

* Trabalho realizado no Registro de Câncer do Ceará e apresentado no II Congresso Paulista de Saúde Pública e I Congresso da ABRASCO, em São Paulo, abril de 1983.

** Coordenador-Executivo do Registro de Câncer do Ceará - Rua Papi Junior, 1222 - 60000 Fortaleza, CE. 
SILVA, M. G. C. da Anos potenciais de vida perdidos segundo causas, em Fortaleza (Brasil), 1978-80.

Rev. Saúde públ., S. Paulo, 18: 108 - 21, 1984.

do duas técnicas diferentes: a primeira, proposta e desenvolvida por Chiang ${ }^{1}$, através das Tábuas de Vida de Múltiplo Decremento, que envolve a utilização de processos estocásticos; e a outra, proposta por Romeder \& $\mathrm{McWhinnie}^{10}$, que independe da utilização da tábua de sobrevivência e não leva em consideração os óbitos ocorridos em idades superiores à vida média da população.

No Brasil, o primeiro método foi trabalhado por Gotlieb ${ }^{3}$ e Ortiz ${ }^{8}$, para o município de São Paulo, e por Silva1 1, 13, para Fortaleza.

Para Meade ${ }^{6}$, a construção de tábuas de vida específicas que permitam estudar e comparar a esperança de vida com várias doenças sucessivamente eliminadas, representa o melhor meio para avaliar a importância relativa de várias doenças para o bem estar econômico de uma população. Entretanto, acrescenta que a construção de tais tábuas é extremamente laboriosa, consumin. do muito tempo, e requer pessoal altamente qualificado e que, assim, a técnica sugerida por Romeder \& McWhinnie ${ }^{10}$ seria uma alternativa de cálculo mais fácil.

Essa técnica, em função da esperança de vida, estabelece uma idade limite, e os óbitos de pessoas acima dessa idade, juntamente com os menores de um ano de idade, são excluídos do estudo. O processo se fundamenta nos anos de vida restantes de cada grupo etário em relação à idade limite pré-estabelecida e esses anos são multiplicados pelo número de mortes de determinada causa do respectivo grupo etário. A soma desses produtos das diversas faixas etárias representa o total de anos potenciais de vida perdidos (APVP).

A técnica de Romeder \& McWhinnie ${ }^{10}$ apresenta ainda outras vantagens, pois pode expressar os APVP para as causas sob forma de números absolutos, taxas, proporção e taxas padronizadas por idade.

Dos estudos de APVP podem ser derivadas outras análises, como as perdas econômicas acarretadas por determinadas causas ou a mensuração do impacto na saúde por conta da adoção de algumas medidas preventivas. Entre essas análises, podem ser citadas a de Millot' ${ }^{6}$ que estudou os efeitos na sobrevida e na esperança de vida e a economia dos recursos por conta de um programa de controle do câncer cérvico-uterino em França, e o de Duarte de Araújo ${ }^{2}$, que estudou a repercussão econômica da mortalidade do município de Salvador, em 1970.

$O$ objetivo deste artigo foi determinar e analisar os anos potenciais de vida perdidos (APVP) para as principais causas de óbitos de Fortaleza, em 1978-80, segundo sexo e idade.

\section{MATERIAL E MÉTODOS}

\section{População}

Os dados da população de 1 a 64 anos, segundo sexo e idade, foram conseguidos junto ao Registro de Câncer do Ceará12. Como o material do estudo se prendeu ao período 1978-80, a população estudada foi a estimada para 01.07 .79 , que correspondia à média do periodo assinalado. Essa estimativa foi obtida pelo método aritmético, por meio de interpolação dos resultados dos cen. sos de 1970 e 1980, e sua distribuição por sexo e grupo etário tomou por base os percentuais censitários de 1970 (Tabela 1).

TABELA 1

População estimada de 1 a 64 anos, segundo sexo e idade, para Fortaleza, em 01.07.79

\begin{tabular}{rrr}
\hline Faixa Etária & Masculino & Feminino \\
\hline $1-4$ & 73.316 & 72.404 \\
$5-9$ & 87.861 & 86.752 \\
$10-14$ & 70.090 & 77.515 \\
$15-19$ & 65.573 & 81.404 \\
$20-24$ & 59.650 & 73.257 \\
$25-29$ & 41.526 & 52.815 \\
$30-34$ & 36.716 & 44.938 \\
$35-39$ & 29.150 & 35.922 \\
$40-44$ & 26.687 & 31.601 \\
$45-49$ & 22.288 & 25.197 \\
$50-54$ & 15.074 & 18.467 \\
$55-59$ & 13.080 & 15.562 \\
$60-64$ & 9.678 & 11.678 \\
\hline $1-64$ & 550.689 & 627.512 \\
\hline
\end{tabular}

Fonte: Registro de Câncer do Ceará ${ }^{12}$. 


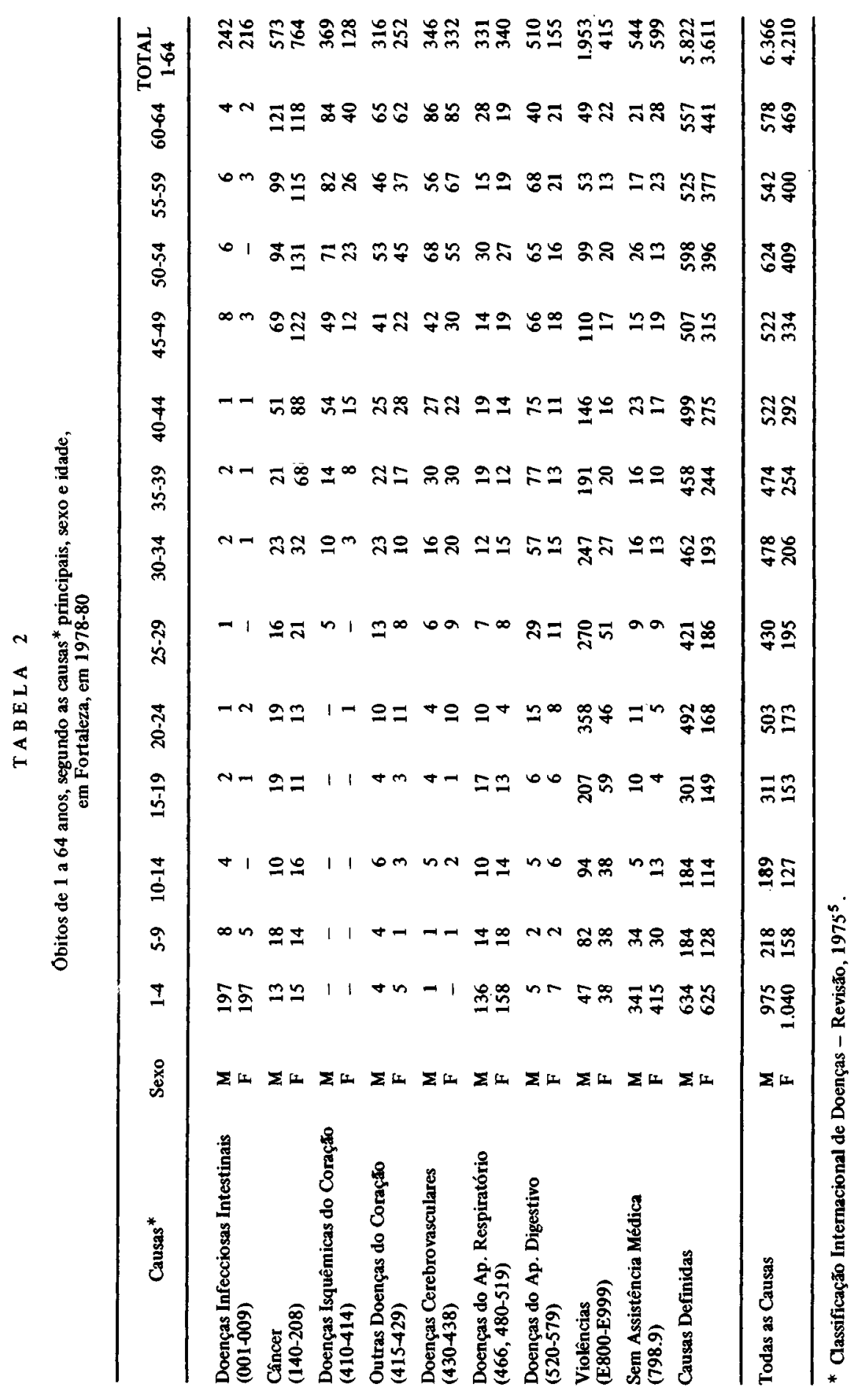


SILVA, M. G. C. da Anos potenciais de vida perdidos segundo causas, em Fortaleza (Brasil), 1978-80. Rev. Saúde públ., S. Paulo, 18: 108 - 21, 1984.

\section{Obitos}

Os dados de obituário empregados neste estudo (Tabela 2) tiveram por fonte a distribuição dos óbitos, segundo sexo e idade, das principais causas ocorridas entre os residentes de Fortaleza, no periodo 1978-80, mostrada por Silva ${ }^{14}$.

Esse autor ${ }^{14}$ verificou que as doenças infecciosas intestinais, o câncer, as outras doenças do aparelho respiratório, as doenças cerebrovasculares, as doenças isquêmicas do coração, as outras doenças do coração e as afecçōes perinatais se destacavam entre as principais causas de morte, em ambos os sexos, e que os acidentes de transportes e os homicídios lograram importantes posições nas mortes masculinas.

Dentre essas causas mais freqüentes, as afecções perinatais foram excluídas porque a técnica adotada no presente trabalho despreza o grupo de menores de um ano de idade, enquanto os acidentes de transportes e os homicídios foram agrupados às demais causas externas, compondo, assim, uma rubrica intitulada de "violências". Além disso, considerando a alta proporção de mortes declaradas, sem causa definida, rotuladas na categoria "sem assistência médica", estas também foram incluídas. Os óbitos sem assistência médica mais aqueles de causas definidas perfazem o total das mortes (todas as causas).

Desse modo, o estudo relacionou, para avaliação, os seguintes agrupamentos de causas ou categoria, com os respectivos códigos da Classificação Internacional de Doenças Revisão 19755 : doenças infecciosas intestinais (001-009), câncer (140-208), doenças is quêmicas do coração (410-414), outras doenças do coração (415-429), doenças cerebrovasculares (430-438), outras doenças do aparelho respiratório (466, 480-519), doenças do aparelho digestivo (520-579), violências (E800-E999) e sem assistência médica (798.9).

Anos Potenciais de Vida Perdidos (APVP)

A técnica de Romeder $\&$ McWhinnie ${ }^{10}$ es tabelece uma idade limite para o cálculo dos APVP com base na vida média da população. No caso de Fortaleza, como a esperança de vida ao nascer, em $1978-80$, era de 59,31 anos nos homens e 65,93 anos nas mulheres ${ }^{14}$, essa idade limite foi convencionada em 65 anos, para ambos os sexos.

0 método de obtenção dos APVP por uma causa específica ou um grupo de causas consiste em somar os produtos do número de mortes em cada idade (entre 1 a 64 anos) pelos anos de vida restantes, até a idade de 65 anos.

A fórmula geral é expressa por:

$$
\operatorname{APVP}={\underset{i=1}{\Sigma}}_{i=1}^{m} a_{i=1}^{m}(m \cdot i \cdot 0,5) d_{i}
$$

onde:

$a_{i}=$ anos de vida restantes até a idade $m$, quando as mortes ocorrem entre as idades $\mathrm{i}$ e it 1 .

$=\mathrm{m}-(\mathrm{i}+0,5)=\mathrm{m} \cdot \mathrm{i}-0,5$

$d_{i}=$ número de mortes entre as idades $i e$ $\mathrm{i}+\overline{\mathrm{i}}$

No caso de Fortaleza, a fórmula fica:

$$
\text { APVP }=\sum_{i=1}^{65} a_{i} d_{i}=\sum_{i=1}^{65}(65 \cdot i \cdot 0,5) d_{i}
$$

Quando efetuados para causas específicas de morte, os APVP são aditivos. Assim, se A e B são duas causas de mortes, então:

APVP $(A+B)=$ APVP $(A)+A P V P(B)$. Isto permite o reagrupamento de causas de mortes sem que se refaçam os cálculos de APVP.

Para o cotejamento com população de tamanhos distintos, os APVP podem ser apresentados sob forma de taxas por 1.000 ou 100.000 habitantes. Em nosso caso, as taxas foram por mil homens ou mulheres, utilizando-se a seguinte fórmula: 
SILVA, M. G. C. da Anos potenciais de vida perdidos segundo causas, em Fortaleza (Brasil), 1978-80.

Rev. Saúde públ., S. Paulo, 18: 108 - 21, 1984.

TAPVP $=\sum_{i=1}^{65} a_{i} d_{i} \times \frac{1.000}{n}$, onde:

$\mathrm{n}=$ número de homens ou mulheres de 1 a 64 anos.

A taxa de APVP pode também ser obtida, segundo grupo etário e sexo, dividindo-se o $a_{j} d_{i}$ dessa faixa e sexo pela população respectiva e multiplicando o resultado por 1.000 .

Outra medida obtida foi a de APVP proporcional, segundo a causa ou grupo de causas e sexo, através da relação:

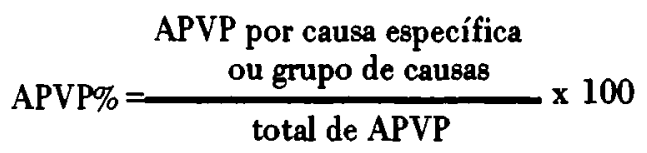

Do mesmo modo que a TAPVP, os APVP
\% também foram mensurados, segundo a faixa de idade e sexo, para os grupos de causas, através da fórmula:

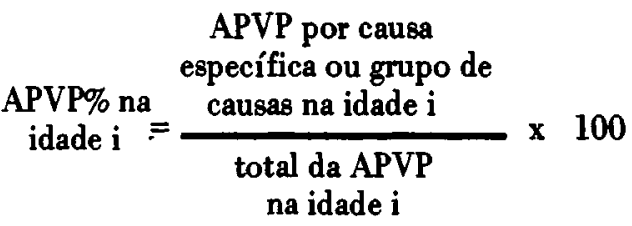

Como exemplo de aplicação da técnica proposta por Romeder \& McWhinnie ${ }^{10}$, a Tabela 3 mostra os APVP por doenças infecciosas intestinais, no sexo masculino, em Fortaleza - 1978-80.

Para cada grupo de causa, foram elaboradas duas tabelas, (uma para cada sexo), conforme a Tabela 3 , composta de seis colunas assim explicitadas:

TABELA 3

Anos potenciais de vida perdidos (APVP) por doenças infecciosas, no sexo masculino, em Fortaleza - 1978-80

\begin{tabular}{cccccc}
\hline $\begin{array}{c}\text { Idade } \\
(1)\end{array}$ & $\begin{array}{c}\mathrm{a}_{\mathrm{i}} \\
(2)\end{array}$ & $\begin{array}{c}\mathrm{d}_{\mathrm{i}} \\
(3)\end{array}$ & $\begin{array}{c}\mathrm{a}_{\mathrm{i}} \mathrm{d}_{\mathrm{i}} \\
(\mathbf{4})\end{array}$ & $\begin{array}{c}\text { TAPVP } \\
(5)\end{array}$ & $\begin{array}{c}\text { APVP\% } \\
(6)\end{array}$ \\
\hline $1-4$ & $\mathbf{6 2}$ & 197 & 12.214 & 55,53 & 20,21 \\
$5-9$ & 57,5 & 8 & 460 & 1,75 & 5,67 \\
$10-14$ & 52,5 & 4 & 210 & 1,00 & 2,12 \\
$15-19$ & 47,5 & 2 & 95 & 0,48 & 0,64 \\
$20-24$ & 42,5 & 1 & 42,5 & 0,24 & 0,20 \\
$25-29$ & 37,5 & 1 & 37,5 & 0,30 & 0,23 \\
$30-34$ & 32,5 & 2 & 65 & 0,59 & 0,42 \\
$35-39$ & 27,5 & 2 & 55 & 0,63 & 0,42 \\
$40-44$ & 22,5 & 1 & 22,5 & 0,28 & 0,19 \\
$45-49$ & 17,5 & 8 & 140 & 2,09 & 1,53 \\
$50-54$ & 12,5 & 6 & 75 & 1,66 & 0,96 \\
$55-59$ & 7,5 & 6 & 45 & 1,15 & 1,11 \\
$60-64$ & 2,5 & 4 & 10 & 0,34 & 0,69 \\
& & & & & \\
\hline Total & - & 242 & $13.471,5$ & 8,15 & 6,81 \\
\hline
\end{tabular}

* Por 1.000 homens

Coluna (1) - Idade - foram colocados os grupos etários compreendidos entre 1 e 64 anos. Excetuando, o primeiro grupo, os demais são compostos de faixas de cinco anos cada. Os intervalos etários foram comuns em todas as tabelas.

Coluna (2) - $a_{i}$ - representa o número de anos de vida restantes na idade i. Esse valor 
SILVA, M. G. C. da Anos potenciais de vida perdidos segundo causas, em Fortaleza (Brasil), 1978-80. Rev. Saúde públ., S. Paulo, 18:108 - 21, 1984.

resulta da diferença observada do ponto médio do grupo etário em relação à idade de 65 anos. Por exemplo, no intervalo de 10 14 anos, o ponto médio é 12,5 anos, que, para 65 anos, indica 52,5 de anos restantes. Esses valores foram também comuns para todos os cálculos de APVP nas diversas causas.

Coluna $(3) \cdot d_{i}$ - é o número de mortes pela causa ou grupo de causas no sexo considerado em cada grupo de idade. Esses números são oriundos da Tabela 2.

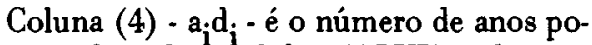
tenciais de vida perdidos (APVP) pela causa ou grupo de causas em cada idade. Resulta da multiplicação dos anos de vida restantes em cada idade $i\left(a_{j}\right)$ pelo número de mortes da(s) causa(s) na idade $i$, no sexo considerado. A soma dos $a_{j} d_{i}$ dá o total de APVP pela(s) causa(s).

Coluna (5) - TAPVP - é a taxa de APVP. Resulta da divisão de cada $a_{i} d_{i}$ nas diversas idades da(s) causa(s) em questão, pela respectiva população da faixa etária e sexo considerados. Como os dados de obituário são pertinentes a um periodo de três anos, a população média do período, vista na Tabela l, precisa ser multiplicada por três para ser obtida a taxa média anual. Os resultados da divisão são multiplicados por 1.000 , de modo que as taxas são expressas por 1.000 homens ou 1.000 mulheres na idade $i$ ou no total de 1 a 64 anos (caso seja efetuada a divisão do total de APVP pela população de 1 a 64 anos), no sexo correspondente.

Coluna (6) - APVP \% - é a proporção de APVP pela(s) causa(s) na idade $i$, no sexo considerado. Esse percentual resulta da divisão do número de $a_{i} d_{i} d a(s)$ causa(s) na idade $i$ pelos $a_{i} d_{i}$ obtidos para todas as causas na correspondente idade $i$ e no mesmo sexo.

\section{RESULTADOS}

Em Fortaleza, no periodo 1978-80, foram sacrificados de sua população, por todas as causas de morte, um total de $335.487,5$ anos potenciais de vida, dos quais 197.942,5 anos atribuídos ao sexo masculino, correspondente a $59,00 \%$ das perdas, e $\mathbf{1 3 7 . 5 4 5}$ anos ao feminino ( $41,00 \%$ das perdas). Em termos populacionais, esses valores signifcaram taxas de 119,82 APVP por 1.000 homens e 73,06 por 1.000 mulheres. A relação masculino/feminino foi 1,44 no número de APVP e 1,64 na taxa de APVP, confirmando a maior perda do sexo masculino (Tabela 4).

No que tange às perdas por causas definidas, o conjunto das violências foi o maior responsável pelos APVP nos dois sexos. Nos homens respondeu por 67.984 APVP, proporcionando cerca de $34,35 \%$ de todas as perdas e taxas de 41,15 por 1.000 , enquanto, nas mulheres, com 15.918,5 APVP, foi de $11,57 \%$ e taxa de 8,46 por 1.000 . Confirmando o exuberante excedente de perdas masculinas, a relação masculino/feminino foi 4,27 no número de APVP e 4,86 na taxa de APVP.

As doenças infecciosas intestinais, a segunda causa de APVP, nos homens e a quinta nas mulheres, contribuíram com 13.471,5 APVP, taxa de 8,15 por 1.000 e $6,81 \%$ dos APVP, nos homens, e 12.796,5 APVP, 6,80 por 1.000 e $9,30 \%$ dos APVP, nas mulheres. A taxa de APVP masculina superou em $20 \%$ a do feminino. As outras doenças do aparelho respiratório, terceira causa de APVP no masculino e segunda no feminino, contribuíram com 13.400 APVP, 8,11 por 1.000 e $6,77 \%$, nos homens, e 14.646 APVP, 7,78 por 1.000 e $10,65 \%$, nas mulheres. $\mathrm{Na}$ seqüência, o câncer, as doenças do aparelho digestivo, as doenças cerebrovasculares, as outras doenças do coração e as doenças isquêmicas do coração marcaram nos APVP, respectivamente, as quinta, quarta, sétima, sexta e oitava posições, nos homens, e as terceira, sétima, quinta, sexta e oitava, nas mulheres.

A relação masculino/feminino das taxas de APVP foi de 3,34 nas doenças do aparelho digestivo e 3,58 nas doenças isquêmicas do coração, enquanto para o câncer foi de 0,85 , sendo o único grupo de causas que mostrou as maiores perdas entre as mulheres.

As mortes sem assistência médica, com 26.884,5 APVP nos homens e 30.695 APVP nas mulheres, destacaram-se porque, em rela- 
SILVA, M. G. C. da Anos potenciais de vida perdidos segundo causas, em Fortaleza (Brasil), 1978-80. Rev. Saride públ, S. Paulo, 18: 108 - 21, 1984.

T A B E L A 4

Anos potenciais de vida perdidos, segundo causa * de morte e sexo, em Fortaleza - 1978-80 ( $n$ ? de anos, taxa ** e percentagem)

\begin{tabular}{|c|c|c|c|c|c|c|}
\hline \multirow{2}{*}{ Causas" } & \multicolumn{3}{|c|}{ Masculino } & \multicolumn{3}{|c|}{ Feminino } \\
\hline & no de anos & Taxas ** & $\%$ & ne de anos & Taxas * * & $\%$ \\
\hline $\begin{array}{l}\text { Doenças Infecciosas In- } \\
\text { testinais }(001-009)\end{array}$ & $13.471,5$ & 8,15 & 6,81 & $12.796,5$ & 6,80 & 9,30 \\
\hline Câncer (140-208) & 10.576 & 6,40 & 5,34 & $14.257,5$ & 7,57 & 10,37 \\
\hline $\begin{array}{l}\text { Doenças Isquêmicas do } \\
\text { coraçăo }(410-414)\end{array}$ & $4.682,5$ & 2,83 & 2,37 & 1.490 & 0,79 & 1,08 \\
\hline $\begin{array}{l}\text { Outras Doenças do Cora- } \\
\text { çâo }(415-429)\end{array}$ & 5.698 & 3,45 & 2,88 & $4.237,5$ & 2,25 & 3,08 \\
\hline $\begin{array}{l}\text { Doenças Cerebrovascula- } \\
\text { res }(430-438)\end{array}$ & $5.139,5$ & 3,11 & 2,60 & 4.870 & 2,59 & 3,45 \\
\hline $\begin{array}{l}\text { Outras Doenças Ap. Res- } \\
\text { piratório }(466,480-519)\end{array}$ & 13.400 & 8,11 & 6,77 & 14.646 & 7,78 & 10,65 \\
\hline $\begin{array}{l}\text { Doenças do Ap. Digesti- } \\
\text { vo }(520-579)\end{array}$ & $=10.932,5$ & 6,62 & 5,52 & 3.664 & 1,98 & 2,70 \\
\hline Violências (E800-E999) & 67.984 & 41,15 & 34,35 & $15.918,5$ & 8,46 & 11,57 \\
\hline $\begin{array}{l}\text { Sem Assistência Médica } \\
\text { (798.9) }\end{array}$ & $26.884,5$ & 16,27 & 13,58 & 30.695 & 16,31 & 22,32 \\
\hline Causas Definidas & 171.058 & 103,54 & 86,42 & 106.850 & 56,76 & 77,68 \\
\hline Todas as Causas & $197.942,5$ & 119,82 & 100,00 & 137,545 & 73,06 & 100,00 \\
\hline
\end{tabular}

* Classificação Internacional de Doenças-Revisão, 19755.

* * Por 1.000 habitantes.

ção aos grupos de causas, forneceram taxas de APVP acima das vistas em todos os gru. pos nas mulheres e somente superada pelo conjunto das violências entre os homens. As mortes não assinadas por médicos equivaleram a $13,58 \%$ e $16,31 \%$ de APVP, nos homens e mulheres, respectivamente, ficando as causas definidas com $86,42 \%$ e $77,68 \%$ (Tabela4).

As taxas de APVP, segundo causas, sexo e idade, estão mostradas na Tabela 5. Para todas as causas as taxas de APVP de um a 4 anos foram altas $(274,83$ por 1.000 homens e 296,85 por 1.000 mulheres); a seguir, essas taxas caíram para 47,56 por 1.000 homens e 34,91 por 1.000 mulheres em 5 a 9 anos, e no grupo seguinte -10 a 14 anos - registraram os valores mais baixos $(47,19$ e 28,67); a partir dos 15 anos, em ambos os sexos, manifestaram tendência ascendente até aos 50.54 anos, com as taxas máximas de 172,48 por 1.000 homens e 92,28 por 1.000 mulheres, e depois, nos dois últimos grupos etários, declinaram. Excetuando a faixa de 1 a 4 anos, nas demais idades as taxas masculinas superaram as femininas, sendo que nos grupos de 15 a 44 anos a relação masculino/feminino vai de 2,12 (40-44 anos) a 3,57 (20-24 anos).

As violências, nos homens, tiveram tendência ascendente com a idade até aos 20-24 anos (taxa de 85,02 por 1.000 ) e a seguir as taxas decresceram e, nas mulheres, não se notou comportamento ascendente ou des- 
SILVA, M. G. C. da Anos potenciais de vida perdidos segundo causas, em Fortaleza (Brasil), 1978-80. Rev. Saúde públ., S. Paulo, 18:108 - 21, 1984.

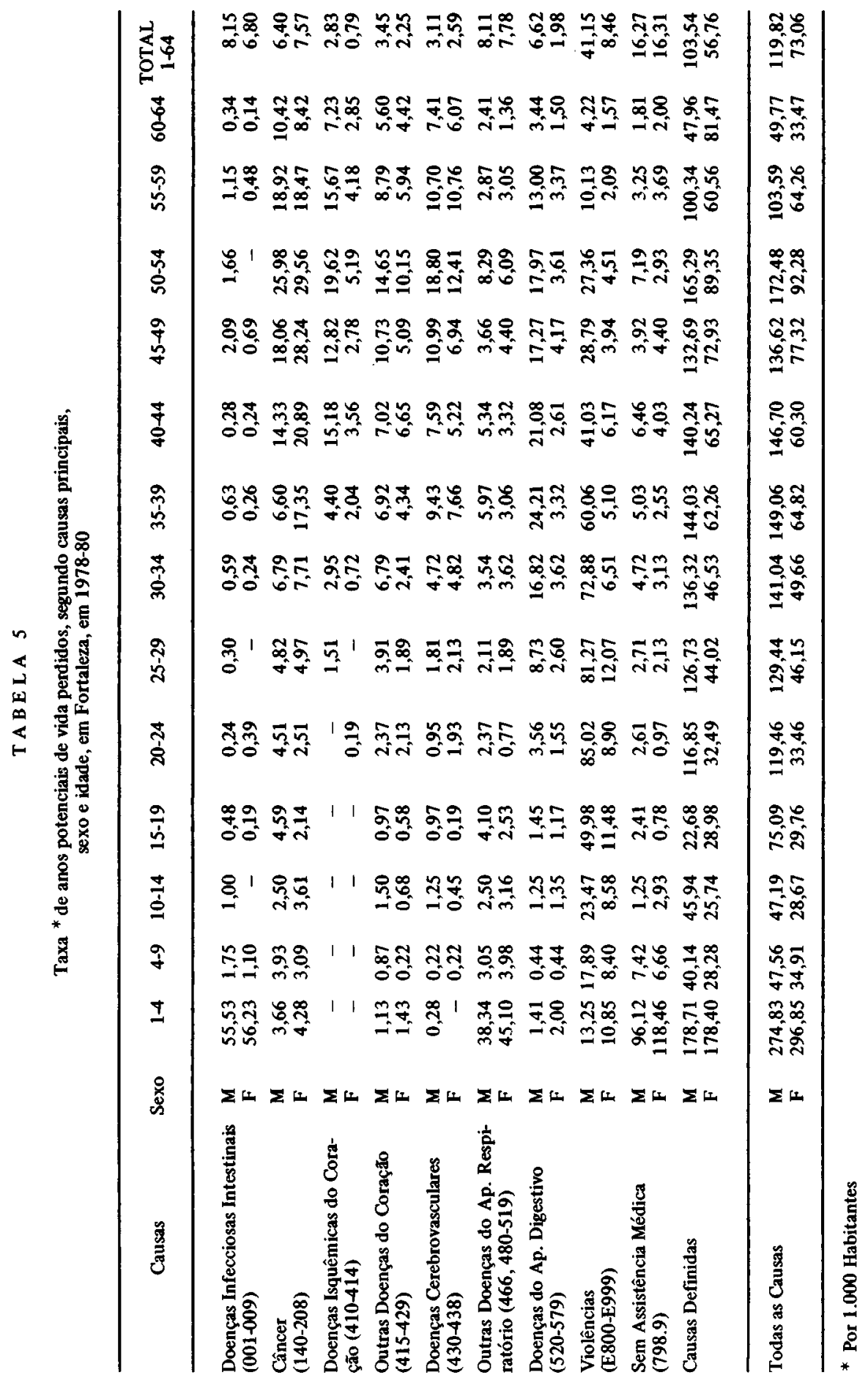


SILVA, M. G. C. da Anos potenciais de vida perdidos segundo causas, em Fortaleza (Brasil), 1978-80. Rev. Saúde públ., S. Paulo, 18: 108 - 21, 1984.

cendente com a idade, mas percebeu-se uma concentração de taxas mais altas entre os grùpos de menores de 30 anos. A relação das taxas de APVP masculino/feminino teve tendência crescente com a idade, até aos 35-39 anos, quando ficou em 11,78. De um modo geral, entre os adultos jovens, foi de aproximadamente 10 vezes maior o risco masculino.

As doenças infecciosas intestinais $e$ as outras doenças do aparelho respiratório mostraram índices altos de APVP apenas no grupo de 1 a 4 anos, sendo similar ao que foi observado entre as mortes sem assistência médica, com destaque para o fato de que nestas a taxa feminina superou a masculina em $23,24 \%$.

0 câncer registrou tendência ascendente com a idade, atingindo o nível mais alto de taxa de APVP no grupo 50.54 anos (25,98 por 1.000 homens e 29,56 por 1.000 mulheres). Dos 25 aos 54 anos, as taxas de APVP foram mais altas entre as mulheres e de 55 a 64 anos, nos homens; abaixo dos 25 anos, as mulheres superaram também nas faixas de 1 a 4 anos e 10 a 14 anos.

As doenças cerebrovasculares, as doenças isquêmicas do coração e as outras doenças do coração retrataram de modo comum tendência ascendente com o avanço da idade, atingindo valores máximos nessas causas, em ambos os sexos, na faixa de 50 a 54 anos.

As doenças do aparelho digestivo revelaram taxas maiores de APVP nos grupos compreendidos entre 30 e 54 anos, principalmente entre os homens, cujas taxas em algumas idades chegaram a ser de sete a oito vezes as observadas nas mulheres.

Entre as causas definidas, excluindo as mortes sem assistência médica, foram notadas as altas taxas no grupo de 1 a 4 anos, mas não em níveis tão elevados como nos de todas as causas, ressaltando a sensível contribuição das mortes declaradas para a taxa de APVP no grupo pré-escolar. Nas demais idades, como as mortes sem assistência médica foram pouco representantivas, suas taxas foram semelhantes às encontradas para todas as causas.
As proporçōes de APVP, segundo causas, sexo e idade, estão apresentadas na Tabela 6. Como já foi referido, a soma das proporç̋es de APVP por causas definidas e sem assistência médica totaliza os $100 \%$ de cada grupo etário. Entre as causas definidas, apenas os grupos de 1 a 4 anos e de 5 a 9 anos demonstraram percentagens inferiores a $85 \%$ pois, às demais idades, nos dois sexos, têm-se niveis acima desse valor. Assim, em conseqüência, as mortes sem assistência médica, em termos de APVP\%, foram muito representativas nos dois grupos assinalados, notadamente entre 1 a 4 anos.

As doenças infecciosas intestinais $e$ as outras doenças do aparelho respiratório revelaram alto peso nos APVP de 1 a 4 anos, nos dois sexos, e nesse grupo, entre as causas definidas, ocuparam as primeira e segunda posiçōes como APVP\%.

As violências arcaram com a maior percentagem de APVP nos grupos compreendidos entre 5 a 54 anos, nos homens, e 5 a 29 anos, nas mulheres. Nos homens, dos 15 aos 29 anos, essa responsabilidade foi cerca de dois terços de todas as perdas, e nas mulheres, de 15 a 19 anos, atingiram um máximo de APVP (38,56\%).

As doenças do aparelho digestivo manifestaram proporções mais altas, nos homens, de 30 a 59 anos, e dos 30 aos 44 anos somente foram superadas pelos índices vistos nas violências.

0 câncer, as doenças cerebrovasculares, as doenças isquêmicas do coração $e$ as outras doenças do coração retrataram tendência crescente com a idade. Dentre essas causas, - câncer destacou-se por ser a principal responsável de APVP\% de 55 a 64 anos, nos homens, e de 30 a 64 anos, nas mulheres.

\section{DISCUSSÃO}

Os números absolutos de APVP oferecem apenas uma idéia bruta da situação, ainda que fácil de entendimento pelos leigos e pú. blico em geral, pois não tomam em consideração a população sob risco, bem como o período de abrangência. $E$ muito natural que grandes populaçóes tenham valores 


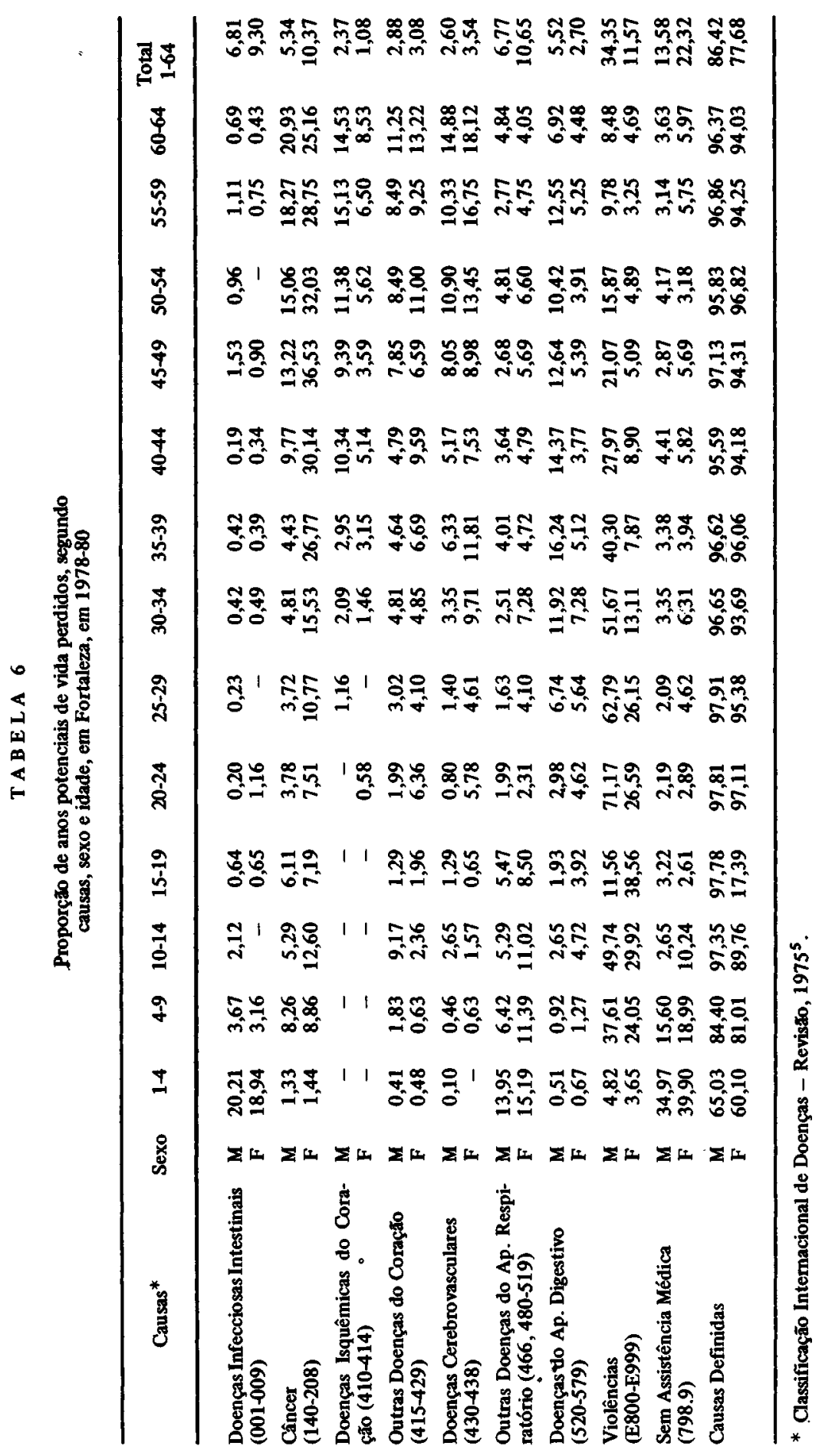


SILVA, M. G. C. da Anos potenciais de vida perdidos segundo causas, em Fortaleza (Brasil), 1978-80. Rev. Saúde públ., S. Paulo, 18:108 - 21, 1984.

mais altos de APVP que comunidades de contingentes mais modestos, assim como num estudo compreendendo dois ou mais anos de uma localidade os resultados de APVP serão maiores que os de somente um ano. Mesmo assim, os números absolutos de APVP mostraram o excedente masculino, e quando expressos sob taxas, ratificaram as maiores perdas masculinas. Esse achado vem ao encontro da sobretaxa de mortalidade masculina vista por Silva ${ }^{14}$.

As violências, por seus altos índices nos APVP, ocuparam uma posição de destaque dentre as principais causas de morte. Sil$\mathrm{va}^{15}$ colocou as violências como importante problema de saúde pública em Fortaleza, em parte, porque foram responsáveis por $12,70 \%$ das mortes masculinas e $3,70 \%$ das femininas, no periodo $1978-80$; se esses percentuais fossem confrontados com as proporções de APVP verificadas aqui $(34,35 \%$ nos homens e $11,57 \%$ nas mulheres) ampliar-se-ia a magnitude das violências como problema de saúde pública que reclama medidas de controle efetivas e urgentes. A ampliação citada é fruto do maior peso das violências entre os adolescentes e adultos jovens do que em outros grupos de doenças.

Romeder e McWhinnie ${ }^{10}$, estudando os APVP na faixa de 1 a 70 anos, no Canadá, em 1974, mostraram que as violências responderam por $38,89 \%$ dos APVP. Vale relatar que a mortalidade proporcional de 1 a 70 anos para alguns tipos de violências tiveram também ampliadas suas proporções em APVP. Assim, os acidentes de trânsito, os outros acidentes e os suicídios, com $8,0 \%, 6,5 \%$ e $3,7 \%$, respectivamente, passaram para $18,2 \%, 12,6 \%$ e $6,4 \%$ de APVP.

As doenças infecciosas intestinais, por sua vez, apesar de lograrem em APVP a $2^{\mathrm{a}}$ posição, nos homens, e a $4^{\mathrm{a}}$, nas mulheres, tiveram declínio de sua importância, pois Sil$\mathrm{va}^{14}$ posicionou-as como a principal causa de morte, na capital cearense, em 1978-80, já que suas taxas foram de 140,49 por 100.000 homens e 96,96 por $100.000 \mathrm{mu}$ lheres e a mortalidade proporcional de $14,86 \%$ nos homens e $14,24 \%$ nas mulheres. Esse decréscimo deve ser fruto da própria técnica da APVP aqui empregada, que despreza as mortes dos menores de um ano. Das 2.472 mortes por doenças infecciosas intestinais, nos homens, $2.195(88,79 \%)$ ocorreram em menores de um ano de idade, e das 1.948 do sexo feminino, 1.689 $(86,70 \%)$ foram nesse grupo infantil, ou seja, pela técnica adotada foi utilizada apenas uma modesta parcela das mortes por doenças infecciosas intestinais. Entretanto, apesar da restrição imposta, as doenças infecciosas intestinais ainda responderam por $6,81 \%$ de APVP nos homens e 9,30\% de APVP nas mu. lheres principalmente por conta do seu alto peso no grupo de 1 a 4 anos, pois, segundo Silva e Bastos ${ }^{16}$, foram a principal causa de mortalidade pré-escolar nos dois sexos.

Da mesma forma, para as outras doenças do aparelho respiratório, foram excluídos $45,22 \%$ dos óbitos masculinos e $39,63 \%$ dos femininos por serem de menores de um ano de idade; contudo, em decorrência de seu peso na faixa de 1 a 4 anos esse grupo de doenças ainda conseguiu se manter em importância nos APVP.

Similarmente, por esse critério de exclusão, não foram computadas 3.248 mortes masculinas e 2.803 femininas de menores de um ano entre aquelas sem assistência médica. Como essas mortes declaradas apresentam um padrão de distribuição por sexo e idade e ao longo do tempo muito semelhante ao das doenças infecciosas intestinais, é muito provável que a parcela maior desses eventos vitais, não atestados por médico, seja por gastroenterites, de modo que os valores de APVP para as doenças infecciosas intestinais estariam subestimados.

Por outro lado, doenças crônico-degenerativas, que atuam mais em idades avançadas, como as isquêmicas do coração, as cerebrovasculares entre outras, têm o seu impacto nos APVP diminuído em função da idade limite. Como se trata de estudar anos potenciais é mais fácil acatar a restrição pelo limite superior com base na vida média da população do que aquela imposta aos óbjtos de menores de um ano de vida.

Romeder e McWhinnie ${ }^{10}$ mostraram uma retração nos valores da mortalidade pro- 
SILVA, M. G. C. da Anos potenciais de vida perdidos segundo causas, em Fortaleza (Brasil), 1978-80. Rev. Saúde públ., S. Paulo, 18:108 - 21, 1984.

porcional quando passam a expressar a porcentagem de APVP, nas doenças crônico-degenerativas.

Das doenças crônico-degenerativas, o câncer, por atingir substanciais segmentos da meia-idade, foi o que obteve as mais altas taxas de APVP (6,40 por 1.000 homens e 7,52 por 1.000 mulheres). Em 1976 , as taxas de APVP pelo câncer em Tailândia, Cingapura e Hong Kong foram, respectivamente, $1,75,9,67$ e 10,93 por 1.000 homens e $2,17,6,12$ e 6,38 por 1.000 mulheres $^{6}$. Nos homens, a taxa de Fortaleza seria maior apenas que a da Tailândia, mas, nas mulheres, seria acima das observadas nesses países.

As taxas de APVP das várias formas de doenças crônico-degenerativas, entre nós, foram inferiores às observadas para o Canadá, em $1974^{10}$.

$\hat{E}$ oportuno ressaltar que o emprego dos APVP é dotado de alto poder discriminatório, pois em região pobre e de baixo nível de saúde as perdas são maiores que as observadas em locais desenvolvidos e de bom ní. vel de saúde, notadamente para as doenças infecciosas e parasitárias 6 .

Em Fortaleza, o câncer mostrou taxa de APVP, nos homens, menor que a observada em doenças infecciosas intestinais, mas, nas mulheres, superou a destas. Silva ${ }^{11},{ }^{13}$, estudando os APVP através das tábuas de vida de múltiplo decremento, encontrou ganhos na esperança de vida ao nascer de 1,41 anos, nos homens e 2,05 anos, nas mulheres, com a eliminação do câncer, e ganhos de 2,82 anos nos homens e 2,29 anos nas mulheres, com a eliminação das doenças infecciosas intestinais como causa de morte.

Os APVP são instrumentos para nortear a escolha de prioridades, mas é preciso ter em consideração a capacidade de redução do dano. Com efeito, mesmo que o câncer revelasse taxas bem mais altas do que as das doenças infecciosas intestinais, estas, como são mais facilmente redutíveis, devem merecer a condição de prioridade.

Para que os estudos de APVP não sejam vistos sob plano teórico ou hipotético, torna-se mais interessante que suas análises sejam efetuadas segundo grupos de causas re- dutíveis, como os que foram empregados por $\mathrm{Ortiz}^{8}$, na capital paulista.

Por fim, é justo enfatizar a necessidade de estudar e analisar os APVP, com o intuito de utilizá-los para o processo do planejamento em saúde, pois, estes, indubitavelmente, constituem relevante instrumento auxiliar à seleção das prioridades a serem atendidas em função dos recursos disponíveis.

\section{CONCLUSÕES}

Com base nos resultados apresentados para Fortaleza conclui-se que:

1. o total de APVP foi de 197.942,5, nos homens, e 137.545, nas mulheres, corres. pondendo a taxas de 119,82 por 1.000 homens e 73,06 por 1.000 mulheres, mostrando um excedente de perdas masculinas;

2. as violências despontaram em primeiro lugar, nos dois sexos, como responsável maior por APVP, ao retratarem, nos homens, perda de 67.984 anos, e taxa de 41,15 por 1.000 , correspondendo a $34,35 \%$ dos APVP, enquanto, nas mulheres, esses valores foram, respectivamente, de $15.915,5,8,46$ e $11,57 \%$;

3. os óbitos sem assistência médica corresponderam a $26.884,5$ anos, 16,27 por 1.000 e $13,58 \%$ de APVP, nos homens, e a 30.695 anos, 16,31 por 1.000 e $22,32 \%$ de APVP, nas mulheres;

4. em ordem de importância em APVP, as principais causas foram as violências, as doenças infecciosas intestinais, as outras doenças do aparelho respiratório, as doenças do aparelho digestivo e o câncer, nos homens, e as violências, as outras doenças do aparelho respiratório, o câncer, as doenças infecciosas intestinais e as doenças cerebrovasculares, nas mulheres;

5. as principais causas de APVP foram as doenças infecciosas intestinais $e$ as outras 
SILVA, M. G. C. da Anos potenciais de vida perdidos segundo causas, em Fortaleza (Brasil), 1978-80. Rev. Saúde públ., S. Paulo, 18:108 - 21, 1984.

doenças do aparelho respiratório, em crianças, as violências, nos adolescentes $e$ adultos jovens, e as doenças crônico-degenerativas, nas últimas faixas etárias.

SILVA, M. G. C. da [Loss of potential years of life, according to cause of death in Fortaleza (Brazil) 1978-1980] Rev. Saúde públ., S. Paulo, 18:108 - 21, 1984.

ABSTRACT: The aim of this study was to determine and analyse the potential years of life lost (PYLL) of the principal causes of death, by sex and age, in Fortaleza, in 1978-80. Death certificates of the period 1978-80, were classified according to cause, sex and age. The technique adopted for PYLL was the one suggested by Romeder and McWhinnie, the age limit of potential life being established at 65 . The results showed that the total of PYLL was $197,942.5$ for males, and 137,545 for females, corresponding to rates of PYLL of 119.82 per 1,000 men and 73.06 per 1,000 women, and that violent deaths, with 41.15 per 1,000 men and 8.46 per 1,000 women, were the main contributory factor in PYLL in both sexes accounting for $34.35 \%$ of male and $11.57 \%$ of female PYLL. By order of importance in PYLL the principal causes were violence, infectious intestinal diseases, diseases of the respiratory system, and cancer, in males, and violence, diseases of the respiratory system, cancer, infectious intestinal diseases and cerebrovascular diseases, in females. The importance of the use of PYIL methodology for Health Planning, as a tool of orientation in the choice of priorities was stressed.

UNITERMS: Mortality. Health indicators. Health planning.

\section{REFERÊNCIAS BIBLIOGRÁFICAS}

1. CHIANG, C. L. Introduction to stochastic process in biostatistics. New York, J. Wiley, 1968.

2. DUARTE DE ARAUJO, J. O custo da doença: repercussão econômica no município de Salvador, BA, Brasil. Rev. Saúde públ., S. Paulo, 9: 155-68, 1975.

3. GOTLIEB, S. L. D. Mortalidade diferencial por causas, São Paulo, 1970: tóbuas de vida de múltiplo decremento. Säo Paulo, 1977. [Tese de doutoramento - Faculdade de Saúde Pública USP]

4. LAURENTI, R. et al. , Aspectos legais dos eventos vitais: sua inflência na demografia. In Encontro Brasileiro de Estudos Populacionais, Rio de Janeiro, 1974. Rio de Janeiro, Fundação IBGE, 1976. p. 12-7.

5. MANUAL da classificação estatística internacional de doenças, lesões e causas de óbitos; Revisão 1975. São Paulo, Centro da OMS para Classificação de Doenças em Portugués, 1978. 2v.

6. MEADE, M.S. Potencial years of life lost in countries of Southeast Asia. Soc. Sci. Med., 14D: 277.81, 1980.
7. MILLOT, M. J. Interet de la prevention du cancer du col de l'uterus à l'occasion de l'exa. men prenuptial. Rev. Epidem. Santé públ., $26(4)$ : 59-69, 1976.

8. ORTIZ, L.P. A mortalidade por causas evitáveis no Estado de São Paulo, 1975/1976. Inf. Demogr., São Paulo, (4): 49-109, 1980.

9. PASTORELO, E.F. A mensuração das condições de saúde nas comunidades. In:Marlet, J.M. et al. Saúde de comunidade. São Paulo, McGraw-Hill do Brasil, 1976. p. 33-59.

10. ROMEDER, J.M. \& MCWHINNIE, J.R. Le développement des années potentielles de vie perdues comme indicateur de mortalité prématurée. Rev. Epidém. Santé publ., 26 (1): 97-115, 1978.

11.SILVA, M.G.C. da Ganhos potenciais em anos de vida com a exclusão dos óbitos por doen. ças infecciosas intestinais, em Fortaleza, no período 1978-80. São Paulo, 1981. [Mímeografado].

12. SILVA, M.G.C. da, coord. Câncer em Fortale$z a: 1978-80$. Fortaleza, Imprensa Universitária UFC, 1982. 
SILVA, M. G. C. da Anos potenciais de vida perdidos segundo causas, em Fortaleza (Brasil), 1978-80. Rev. Saúde públ., S. Paulo, 18:108 - 21, 1984.

13. SILVA, M.G.C. da Câncer em Fortaleza: morbidade e mortalidade no periodo de 1978-80. Fortaleza. Imprensa Oficial do Ceará, 1982. [Dissertação de Mestrado - Faculdade de Saúde Pública USP]

14.SILVA, M.G.C. da Situação de saúde em Fortaleza: análise através da mortalidade ém 1978 . 80. Fortaleza, Imprensa Oficial do Ceará, 1982.
15.SILVA, M.G.C. da Mortalidade por violências em Fortaleza, em 1978-80. Rev. Fac. Méd. Univ. Fed. Ceard. [no prelo]

16.SILVA, M.G.C. da \& BASTOS, M.F.A. Mortalidade pré-escolar em Fortaleza, em 1978-80. Ceard méd. [no prelo]

Recebido para publicação em 26/06/1983. Aprovado para publicação em 21/12/1983. 